\title{
Partial synchronization and spontaneous spatial ordering in coupled chaotic systems
}

\author{
Ying Zhang, ${ }^{1,2}$ Gang Hu, ${ }^{3,1}$ Hilda A. Cerdeira, ${ }^{4}$ Shigang Chen, ${ }^{2}$ Thomas Braun, ${ }^{5}$ and Yugui Yao ${ }^{6, *}$ \\ ${ }^{1}$ Department of Physics, Beijing Normal University, Beijing 100875, China \\ ${ }^{2}$ LCP, Institute of Applied Physics and Computational Mathematics, P.O. Box 8009(26), Beijing 100088, China \\ ${ }^{3}$ Chinese Center for Advanced Science and Technology (World Laboratory), Beijing 8730, China \\ ${ }^{4}$ The Abdus Salam International Centre for Theoretical Physics, P.O. Box 586, 34100 Trieste, Italy \\ ${ }_{5}^{5}$ Instituto de Fisica, Universidade Federal do Rio Grande do Sul, Caixa Postal 15051, 91501-970 Porto Alegre, RS, Brazil \\ ${ }^{6}$ SKLSP, Institute of Physics \& Center for Condensed Matter Physics, P.O. Box 603-40, Beijing 100080, China
}

(Received 25 April 2000; revised manuscript received 31 October 2000; published 24 January 2001)

\begin{abstract}
A model of many symmetrically and locally coupled chaotic oscillators is studied. Partial chaotic synchronizations associated with spontaneous spatial ordering are demonstrated. Very rich patterns of the system are revealed, based on partial synchronization analysis. The stabilities of different partially synchronous spatiotemporal structures and some dynamical behaviors of these states are discussed both numerically and analytically.
\end{abstract}

DOI: 10.1103/PhysRevE.63.026211

\section{INTRODUCTION}

Synchronization of coupled chaotic systems has recently become a topic of great interest [1], with the intent of realistically modeling spatially extended systems and even turbulent systems, and with the belief that dominant features of the underlying constituents of extended systems will be retained in such simple models. For this intention, coupled systems with local interactions are of significance. Complete synchronization (CS) of chaotic oscillators has been described theoretically and observed experimentally $[1,2]$. Recently, partial synchronization (PS), where some of the subsystems synchronize with each other and others do not, is one of the most important aspects after the complete synchronization is broken. PS of chaotic oscillators has been extensively investigated in globally coupled systems, where no space structure can be involved [3]. Very recently, PS in locally coupled systems has been revealed [4], in which, however, only three subsystems are involved and the nonsymmetric coupling plays the key role in producing partial synchronization. To our knowledge, partial synchronizations associated with the spontaneous symmetry breaking and with different spatial structures in locally and symmetrically coupled chaotic systems have not yet been systematically investigated. However, this is obviously a very important direction.

In this paper, we study PS in symmetrically and locally coupled chaotic oscillators. Our basic model is the chain of coupled identical Rossler oscillators [5] with nearestneighbor diffusive coupling. It can be written as a set of ordinary differential equations

$$
\begin{gathered}
\dot{x}_{i}=-y_{i}-z_{i}+\varepsilon\left(x_{i-1}+x_{i+1}-2 x_{i}\right), \\
\dot{y}_{i}=x_{i}+a y_{i}+\varepsilon\left(y_{i-1}+y_{i+1}-2 y_{i}\right), \\
\dot{z}_{i}=b+z_{i}\left(x_{i}-c\right)+\varepsilon\left(z_{i-1}+z_{i+1}-2 z_{i}\right), \\
x_{N+1}=x_{1}, \quad y_{N+1}=y_{1}, \quad z_{N+1}=z_{1},
\end{gathered}
$$

\footnotetext{
*Author to whom correspondence should be addressed. Email address: ygyao@aphy.iphy.ac.cn
}

PACS number(s): 05.45.Jn, 05.45.Xt

where we take $a=0.175, b=0.4$, and $c=8.5$, which yield a chaotic state of the system, and $i=1,2, \ldots, N$ represents the spatial location of oscillators in the lattice and $\varepsilon$ is the coupling coefficient. Specifically, we focus on the analysis of $N=6$, a direct extension to arbitrary $N$ will be briefly discussed in the conclusion. Due to the structure of the model, the oscillators have the following symmetries. First, the system is invariant against the change between clockwise direction and counterclockwise direction due to the symmetric coupling. Second, Eqs. (1) satisfy spatial permutation symmetry, i.e., they are invariant by the exchange $\vec{r}_{i} \leftrightharpoons \vec{r}_{j}$. Any breaking from these symmetries of the system state must happen spontaneously [6].

Here, we are concerned with the patterns induced by different kinds of PS in the lattice. Imagine, we have now six seats, sitting on a ring in the order of $i=1,2, \ldots, 6$ with 7 being identical to 1 ; each seat has a certain type of oscillation (suppose totally $m \leqslant 6$ types of motions are acceptable), and different seats can have the same type of oscillation. The question is which arrangements for the six seats are acceptable, i.e., which spatial ordering and spatiotemporal patterns can be observed? These patterns comply with their special spatial dynamics in smooth invariant submanifold of lower dimension than that of the full phase space with $6 \times 3$ dimension $(6 \times 3 D)$ (the submanifold is invariant in the sense: any orbit originating in the submanifold stays there forever [7]). The stability of these spatial solutions depend on the dynamics of infinitesimal perturbations that are transverse to their invariant submanifold. We discuss each of the possible PS solutions in the next section.

The paper is arranged as follows. In Sec. II, we find all the possible partially synchronized spatial structures by theoretical analysis. Both their dynamic equations defined in the invariant submanifolds and their instability conditions are deduced. In Sec. III, partially synchronous states and their transverse stabilities are discussed in numerical computation. The actual stable states of the original coupled oscillators are presented in Sec. IV. On the basis of these we have found about all the possible and actual PS structural states in the six coupled chaotic oscillators; we come to obtain a global information about the dynamics of the coupled system in full 
space described by Eqs. (1). The last section presents a brief discussion on the generalization and application of the findings in this paper.

\section{THEORETICAL ANALYSIS OF PARTIALLY SYNCHRONIZED SPATIAL STRUCTURES}

The case $m=1$ is the trivial case of complete synchronization, and has been studied extensively in the previous literatures [8]. So, we start from $m=2$. In this case, the six oscillators are divided into two sets. The motion of the oscillators in a set is identical; we represent one by $a$, and the other by $b$. There are two and only two topologically distinct patterns, one is $\{a b a b a b\}$, and the other is $\{a a b a a b\}$. For instance, the state $\{a a a b b b\}$ can never occur unless $a=b$, because the $a$ and $b$ sites (i.e., $a_{2}, b_{2}$ in $a_{1} a_{2} a_{3} b_{1} b_{2} b_{3}$ ) have dynamics different from the side $a$ and $b\left(a_{1}, b_{3}\right.$ and $\left.b_{1}, b_{3}\right)$ if $a \neq b$ due to the different couplings [see Eqs. (1) for the dynamics].

Given $\{a b a b a b\}$, the $2 \times 3 D$ partially synchronous invariant submanifold is defined by $\vec{r}_{1}=\vec{r}_{3}=\vec{r}_{5}$ and $\vec{r}_{2}=\vec{r}_{4}$ $=\vec{r}_{6}$. The full phase space with $6 \times 3 D$ can be regarded as a direct product of the invariant subspace of $2 \times 3 D$ and its transverse remainder. Under this consideration, we can make the following transformation:

$$
\begin{aligned}
& \vec{R}_{1}=\vec{r}_{1}+\vec{r}_{3}+\vec{r}_{5}, \quad \vec{R}_{2}=\vec{r}_{2}+\vec{r}_{4}+\vec{r}_{6}, \\
& \vec{R}_{3}=-2 \vec{r}_{1}+\vec{r}_{3}+\vec{r}_{5}, \quad \vec{R}_{4}=\vec{r}_{3}-\vec{r}_{5}, \\
& \vec{R}_{5}=\vec{r}_{2}-2 \vec{r}_{4}+\vec{r}_{6}, \quad \vec{R}_{6}=\vec{r}_{2}-\vec{r}_{6}, \\
& \vec{R}_{i}=\left(X_{i}, Y_{i}, Z_{i}\right), \quad i=1,2, \ldots, N .
\end{aligned}
$$

In the given invariant submanifold, the motion can be described by only two coordinates $\vec{R}_{1}$ and $\vec{R}_{2}$, and the dynamics of $\{a b a b a b\}$ on the invariant subspace is governed by the equations

$$
\begin{gathered}
\dot{X}_{1}=-Y_{1}-Z_{1}+2 \varepsilon\left(X_{2}-X_{1}\right), \\
\dot{Y}_{1}=X_{1}+a Y_{1}+2 \varepsilon\left(Y_{2}-Y_{1}\right), \\
\dot{Z}_{1}=3 b+\frac{1}{3} Z_{1} X_{1}-c Z_{1}+2 \varepsilon\left(Z_{2}-Z_{1}\right), \\
\dot{X}_{2}=-Y_{2}-Z_{2}+2 \varepsilon\left(X_{1}-X_{2}\right), \\
\dot{Y}_{2}=X_{2}+a Y_{2}+2 \varepsilon\left(Y_{1}-Y_{2}\right), \\
\dot{Z}_{2}=3 b+\frac{1}{3} Z_{2} X_{2}-c Z_{2}+2 \varepsilon\left(Z_{1}-Z_{2}\right) .
\end{gathered}
$$

The stability of $\{a b a b a b\}$ PS state depends on the infinitesimal perturbations in its transverse remainder subspace, and the dynamics of the perturbations is governed by

$$
\begin{gathered}
\delta \dot{X}_{3}=-\delta Y_{3}-\delta Z_{3}+\varepsilon\left(-2 \delta X_{3}-\delta X_{5}\right), \\
\delta \dot{Y}_{3}=\delta X_{3}+a \delta Y_{3}+\varepsilon\left(-2 \delta Y_{3}-\delta Y_{5}\right),
\end{gathered}
$$

$$
\begin{gathered}
\delta \dot{Z}_{3}=\frac{1}{3} Z_{1} \delta X_{3}+\frac{1}{3} X_{1} \delta Z_{3}-c \delta Z_{3}+\varepsilon\left(-2 \delta Z_{3}-\delta Z_{5}\right), \\
\delta \dot{X}_{4}=-\delta Y_{4}-\delta Z_{4}+\varepsilon\left(-2 \delta X_{4}+\delta X_{6}\right), \\
\delta \dot{Y}_{4}=\delta X_{4}+a \delta Y_{4}+\varepsilon\left(-2 \delta Y_{4}+\delta Y_{6}\right), \\
\delta \dot{Z}_{4}=\frac{1}{3} Z_{1} \delta X_{4}+\frac{1}{3} X_{1} \delta Z_{4}-c \delta Z_{4}+\varepsilon\left(-2 \delta Z_{4}+\delta Z_{6}\right), \\
\delta \dot{X}_{5}=-\delta Y_{5}-\delta Z_{5}+\varepsilon\left(-2 \delta X_{5}-\delta X_{3}\right), \\
\delta \dot{Y}_{5}=\delta X_{5}+a \delta Y_{5}+\varepsilon\left(-2 \delta Y_{5}-\delta Y_{3}\right), \\
\delta \dot{Z}_{5}=\frac{1}{3} Z_{2} \delta X_{5}+\frac{1}{3} X_{2} \delta Z_{5}-c \delta Z_{5}+\varepsilon\left(-2 \delta Z_{5}-\delta Z_{3}\right), \\
\delta \dot{X}_{6}=-\delta Y_{6}-\delta Z_{6}+\varepsilon\left(-2 \delta X_{6}+\delta X_{4}\right), \\
\delta \dot{Y}_{6}=\delta X_{6}+a \delta Y_{6}+\varepsilon\left(-2 \delta Y_{6}+\delta Y_{4}\right), \\
\delta \dot{Z}_{6}=\frac{1}{3} Z_{2} \delta X_{6}+\frac{1}{3} X_{2} \delta Z_{6}-c \delta Z_{6}+\varepsilon\left(-2 \delta Z_{6}+\delta Z_{4}\right),
\end{gathered}
$$

where $\left(\vec{R}_{1}(t), \vec{R}_{2}(t)\right)$ is a possible solution of $\{a b a b a b\}$ obtained by the integration of Eqs. (3). The stability of the state $\left(\vec{R}_{1}(t), \vec{R}_{2}(t)\right)$ depends on the largest Lyapunov exponent (LE) of Eqs. (4),

$$
\Lambda=\lim _{t \rightarrow \infty} \frac{1}{t} \ln [\delta(t) / \delta(0)],
$$

where

$$
\delta(t)=\sqrt{\sum_{j=3}^{6}\left\{\left[\delta X_{j}(t)\right]^{2}+\left[\delta Y_{j}(t)\right]^{2}+\left[\delta Z_{j}(t)\right]^{2}\right\}} .
$$

From the discussion above, the computation procedure is very clear. Given certain possible PS solution, first we perform a space transformation into two subspaces, one of which is the invariant submanifold corresponding to the pattern solution; the other corresponds to its transverse remainder. Second, we consider the dynamics of infinitesimal perturbations that are transverse to the invariant submanifold, and calculate the largest LE for the perturbations, then the stability of the PS solution is determined. Following the same procedure, we can analyze all other PS solutions of our system described by Eqs. (1).

For the $\{a a b a a b\}$ pattern, the $2 \times 3 D$ invariant synchronization submanifold is defined by $\vec{r}_{1}=\vec{r}_{2}=\vec{r}_{4}=\vec{r}_{5}$ and $\vec{r}_{3}$ $=\vec{r}_{6}$. The full phase space with $6 \times 3 \mathrm{D}$ can be built up by the invariant subspace and its transverse remainder. Under this consideration, we perform the transformation

$$
\begin{gathered}
\vec{R}_{1}=\vec{r}_{1}+\vec{r}_{2}+\vec{r}_{4}+\vec{r}_{5}, \quad \vec{R}_{2}=\vec{r}_{3}+\vec{r}_{6}, \\
\vec{R}_{3}=\vec{r}_{1}-\vec{r}_{2}-\vec{r}_{4}+\vec{r}_{5}, \quad \vec{R}_{4}=\vec{r}_{2}-\vec{r}_{4}, \\
\vec{R}_{5}=\vec{r}_{1}-\vec{r}_{5}, \quad \vec{R}_{6}=\vec{r}_{3}-\vec{r}_{6}, \\
\vec{R}_{i}=\left(X_{i}, Y_{i}, Z_{i}\right), \quad i=1,2, \ldots, N,
\end{gathered}
$$


and derive the equations for the evolution of the system on the $\left(\vec{R}_{1}, \vec{R}_{2}\right)$ synchronous invariant submanifold, and the equations for determining the transverse stability in the same manner as Eqs. (3) and (4).

If $m=3$, there are also two topologically different patterns $\{a b b a c c\}$ and $\{a b c a b c\}$ (note, the state $\{a a b b c c\}$ can never occur by the same reason of the forbiddance of $\{a a a b b b\}$ structure). For $m=4$, we have a unique PS state, i.e., the $\{a b c b a d\}$ state. The detailed discussion on the spatial transformations, the equations on the submanifolds of these PS states, and the corresponding linear dynamics transversal to these submanifolds are given in the Appendix.

There is no pattern for $m=5$ with six oscillators, and $m$ $=6$ corresponds to the case of no kind of synchronization. Therefore, we have learned that, apart from the completely synchronized and fully desynchronized solutions, the system of coupled six Rossler oscillators has five kinds of nontrivial PS solutions; they are $\{a b a b a b\},\{a a b a a b\},\{a b b a c c\}$, $\{a b c a b c\}$, and $\{a b c b a d\}$. Their instabilities are determined by their largest conditional LE, $\Lambda$, transverse to the corresponding partially synchronized manifolds.

\section{PARTIALLY SYNCHRONIZED STATES AND THEIR TRANSVERSE STABILITIES}

After deriving all the equations for the PS solutions on various synchronous invariant submanifolds and for the stabilities of these solutions, we can now numerically compute all these solutions and specify their stabilities. For doing this, we compute two kinds of LE's. First, we calculate the two largest LE's in the invariant submanifolds to classify the PS solutions, i.e., to distinguish periodic solution (one zero LE and one negative), quasiperiodic motion (two zero LE's), and chaotic state (at least one positive LE). Second, we compute the largest transverse LE, $\Lambda$, of the corresponding PS solution for determining its stability, i.e., negative (positive) $\Lambda$ indicates the local stability (instability) of this PS state.

We start with $m=1$, the CS state. The two largest LE's in the invariant subspace, $L 1$ and $L 2$, and the largest transversal LE, $\Lambda$, are plotted versus the coupling parameter $\varepsilon$ in Figs. 1(a,b), respectively. From Fig. 1, it is shown that there is only a single synchronous chaotic solution for this trivial case, the chaotic state of a single Rossler oscillator. This complete synchronous chaos is stable for $\varepsilon>0.087$ (called as CSC) since $\Lambda<0$ there, and it is unstable otherwise.

Next, we come to the two PS cases for $m$ $=2:\{a b a b a b\}$ and $\{a a b a a b\}$. For $\{a b a b a b\}, L 1, L 2$ and $\Lambda$ are presented in Figs. 2(a,b), respectively. $L 1$ and $L 2$ are obtained from Eqs. (3) in the corresponding subspace, while $\Lambda$ comes from the computation of Eqs. (4). From Fig. 2(b) one can find two stability regions. One region with $\varepsilon$ $>0.087$ corresponds to a chaotic ababab motion degenerating to the CSC $(a=b)$; the other region is around $\varepsilon=0.2$ where we find stable partially synchronous periodic motion (PSP). In Figs. 2(c,d) we do the same as Figs. 2(a,b) with the $\{a a b a a b\}$ partially synchronous structure being considered, and we observe also two stable regions. One region with $\varepsilon$ $>0.087$ corresponds again to the CSC state, and the other around $\varepsilon \approx 0.0075$ allows stable PSP $\{a a b a a b\}$ motion.

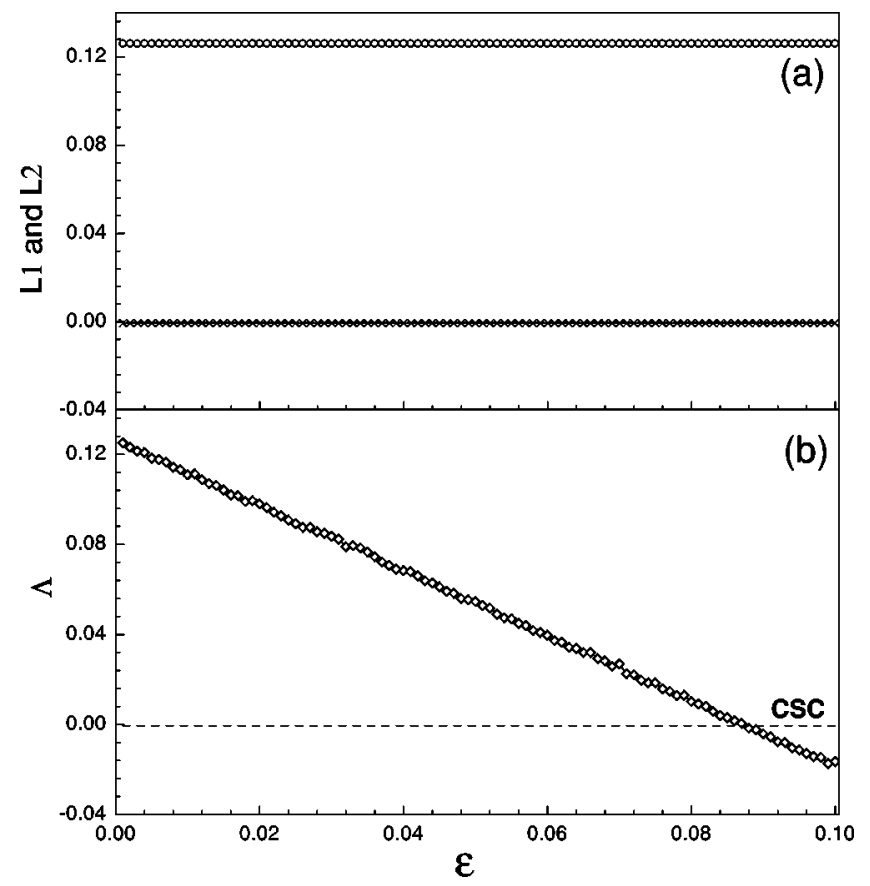

FIG. 1. (a) The largest Lyapunov exponents $L 1$ and $L 2$ versus coupling parameter $\varepsilon$ in the submanifold $\{$ aaaaaa $\}(m=1)$. (b) The transversal largest Lyapunov exponent $\Lambda$ versus $\varepsilon$ corresponding to (a).

In Fig. 3 we do the same as Fig. 2 by having $m=3$ and $\{a b b a c c\}$ and $\{a b c a b c\}$ spatial structures considered, respectively. For the case of $\{a b b a c c\}$, new types of solutions appear. Figure 3 shows that, in addition to the stable periodic states for small $\varepsilon$, there are other periodic and chaotic states keeping stable for $0.059<\varepsilon<0.087$. Partially synchronous chaotic state is called PSC. For $\{a b c a b c\}$, one can find PSP

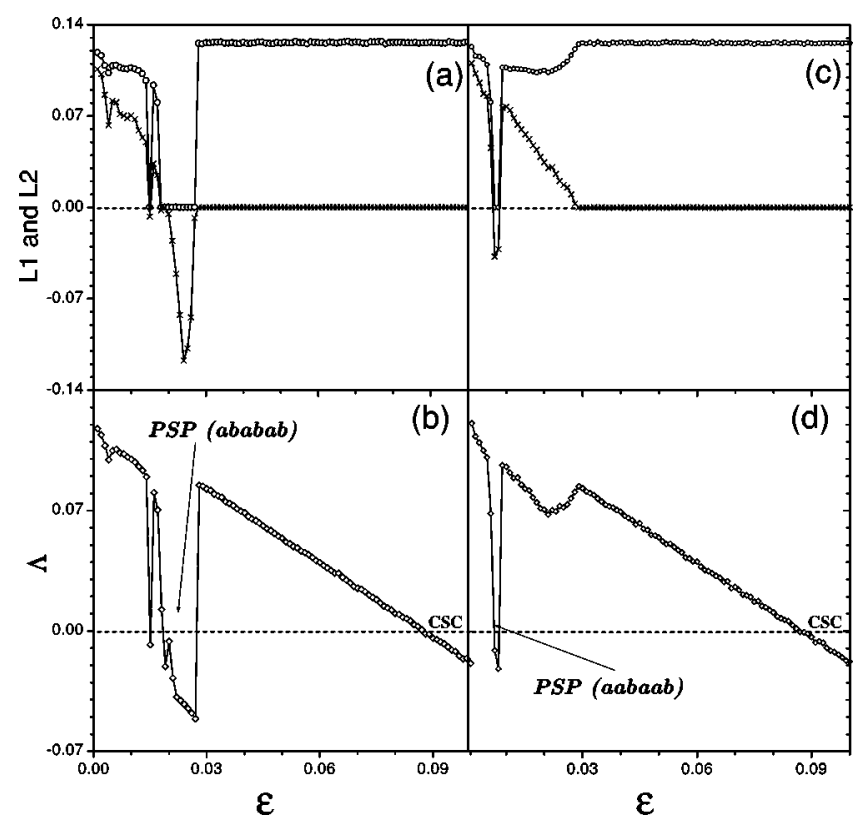

FIG. 2. The same as Fig. 1 with (a) and (b) $\{a b a b a b\}$ $(m=2)$; (c) and (d) $\{a a b a a b\}(m=2)$. 


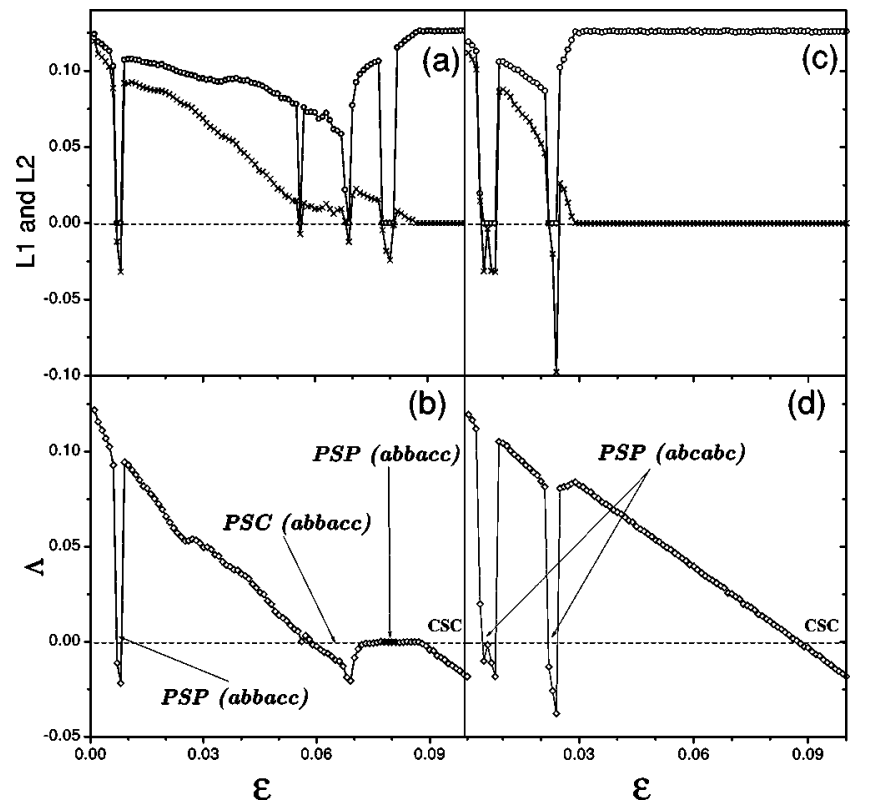

FIG. 3. The same as Fig. 1 with (a) and (b) $\{a b b a c c\}$ $(m=3)$; (c) and (d) $\{a b c a b c\}(m=3)$.

only for relatively small $\varepsilon$, similar to the case of $m=2$.

For $m=4$, we have a single spatial structure $\{a b c b a d\}$ only. The corresponding Lyapunov exponents are plotted in Fig. 4. The curves of $L 1, L 2$ and $\Lambda$ in Figs. 4(a,b) show that the temporal behavior in this submanifold of this spatial structure is rich. In particular, we can find stable partially synchronous quasiperiodic motion in the region $0.060<\varepsilon$ $<0.068$, which can bifurcate to partially synchronous chaos with the same $a b c b a d$ structure by increasing $\varepsilon$. Moreover, we find a stable partially synchronous (in the way of

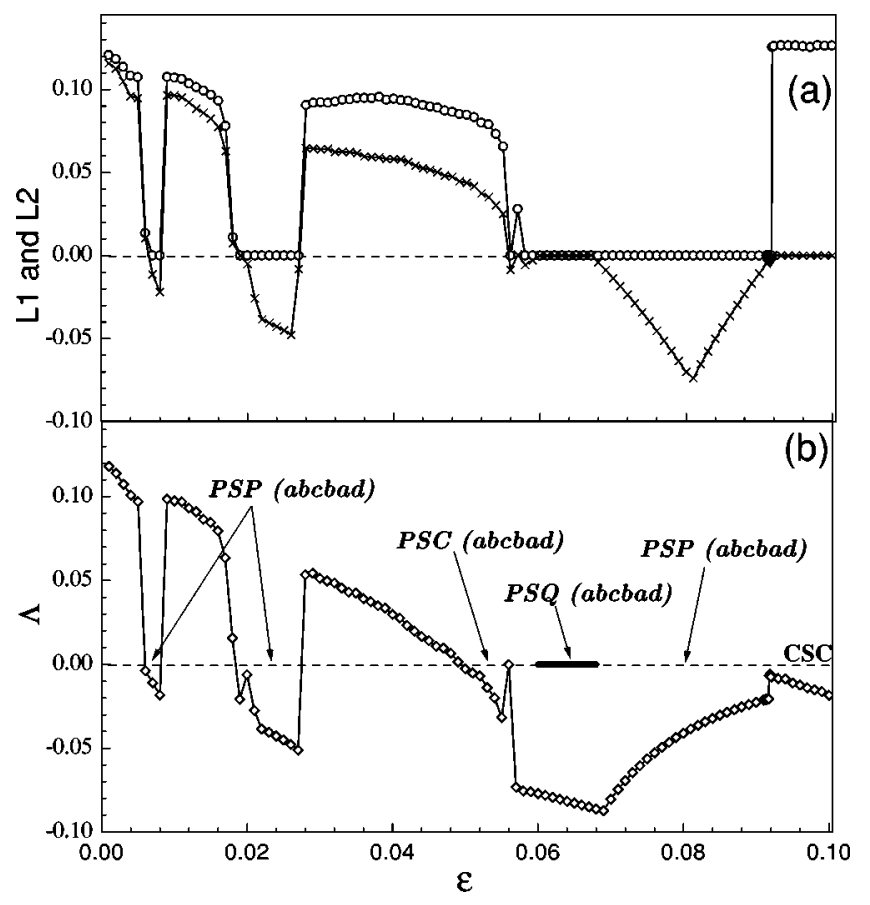

FIG. 4. The same as Fig. 1 with $\{a b c b a d\}(m=4)$. abcbad) periodic state for $0.087<\varepsilon<0.091$, which coexists with the completely synchronous chaotic state.

\section{THE ACTUAL STATE OF THE ORIGINAL COUPLED OSCILLATORS}

All the results in Figs. 1-4 are from the dynamics of various invariant subspaces of the system and their transversal remainders. In this section, we investigate their corresponding actual states by directly computing the original system Eqs. (1). From Figs. 1-4, we note that there are multiple stable solutions of CS and PS coexisting in some coupling ranges. So, in order to surely obtain certain PS solutions, we have to restrict the initial states in the vicinity of the wanted pattern; the problem about the attracting basins of the PS states will be discussed later in the conclusion section. In the following, we take the same order as the last section and present the actual states for all the PS patterns one by one.

For $m=1$, the completely synchronous state takes the chaotic orbit of a single Rossler oscillator, and all oscillators have an identical trajectory.

For the stable $\{a b a b a b\}$ structure both $a$ and $b$ oscillators have the same period- 2 orbit [Fig. 5(a)], but they take different phases on the orbit [see the two circles in Fig. 5(a) and also see Fig. 5(b)]. The oscillators $a$ and $b$ in the $\{a a b a a b\}$ structure become slightly different [see Fig. 5(c)], the phase relation between $a$ and $b$ for this structure is shown in Fig. $5(d)$.

The characteristic features of the $\{a b b a c c\}$ PSP state, shown in Fig. 6, are particularly interesting. Both $b$ and $c$ oscillators take the same period-6 orbit [see Fig. 6(a)] while the phases of $b$ and $c$ are different [see Fig. 6(b)]. However, the oscillators $a$ have a period-3 orbit [see Fig. 6(c)]. How can a period-3 orbit exist under the interactions of period-6 signals? The reason is that both $x_{b}(t)$ and $x_{c}(t)$ have period-6 orbits while the total coupling to an oscillator $a$, $\varepsilon\left[x_{b}(t)+x_{c}(t)\right]$, can become of period-3 after summation, which can be seen in Fig. 6(d). There is also a chaotic $\{a b b a c c\}$ PSC state; the spatiotemporal behavior is not striking and we do not show it here. We find two kinds of $\{a b c a b c\}$ PSP states, and one example is exhibited in Fig. 7. For the case in Fig. 7, the $a, b$, and $c$ oscillators take the same period-3 orbit [see Fig. 7(a)], while they array in $a b c$ with $T / 3$ ( $T$ is the period) phase difference [see the circles indicated in Fig. 7(a), and the phase relation in Fig. 7(b) where the thick and thin curves are symmetric against the diagonal line]. The other $\{a b c a b c\}$ PSP case considered is similar to that of Fig. 6 . The existence of both period-2 and period-4 orbits and the reasoning for this seemly peculiar phenomenon are exactly the same as of Fig. 6.

Figure 8 shows the $\{a b c b a d\}(m=4)$ partial synchronous periodic (PSP), partial synchronous quasiperiodic (PSQ), and partial synchronous chaotic (PSC) states, respectively. The $a$ and $b$ periodic oscillators have the same orbit, but take different phases. Similar spatial relations can be also observed for the PSQ [Figs. 8(c,d)] and PSC [Figs. 8(e,f)] states.

In all the figures from Fig. 5 to Fig. 8, we show the dynamic behavior of various stable configurations of the system. It is interesting to investigate how some of these patterns appear via different bifurcations and symmetry break- 

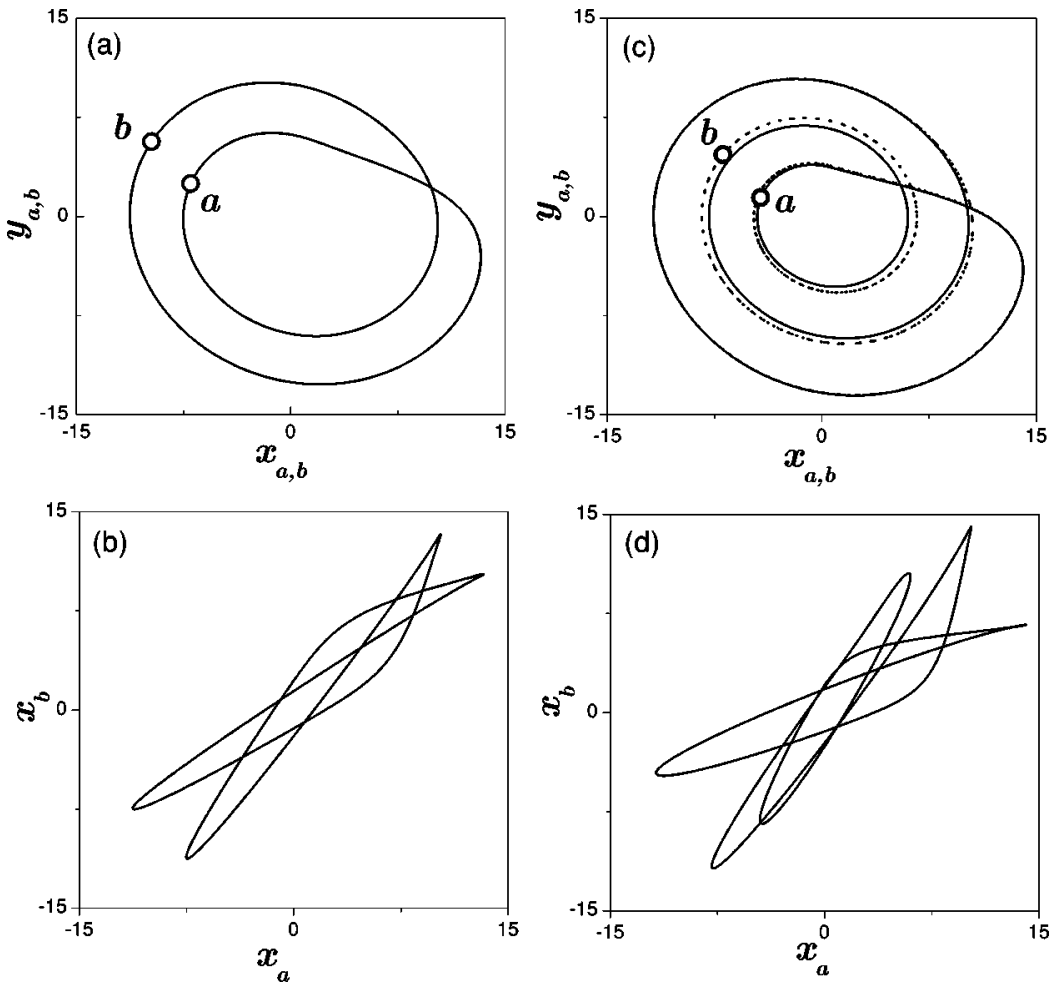

FIG. 5. (a) and (b) Periodic oscillations for the pattern of $\{a b a b a b\}$ with $\varepsilon=0.025$. (a) $x_{a, b}$ vs $y_{a, b}$, (b) $x_{a}$ vs $x_{b}$. (c) and (d) Periodic oscillations for the pattern of $\{a a b a a b\}$ with $\varepsilon$ $=0.008$. (c) $x_{a}$ vs $y_{a}$, solid curve; $x_{b}$ vs $y_{b}$, dashed curve. (d) $x_{a}$ vs $x_{b}$. The letters $a$ and $b$ in the figure indicate the positions of the $a$ and $b$ sites at an arbitrary instant. This notation also is used in Figs. 5-8. ings by varying the control parameters. For this sake, we start from the CSC state of $\varepsilon>\varepsilon_{c}$, and perform our numerical simulation by continually reducing $\varepsilon$ (the step for $\varepsilon$ change is $\Delta \varepsilon=0.001$ ). For each $\varepsilon$ we run the system (1) by taking the ending state for the previous coupling as the initial state for the current coupling. Small noise is used to exclude any unstable states. In Fig. 9, we plot the three largest
Lyapunov exponents of the system state realized in the above process versus the coupling intensity $\varepsilon$. We find, at $\varepsilon=\varepsilon_{c}$ $\approx 0.087$, a translational symmetry breaking (the orientation symmetry is still kept) occurs, which brings the system from CSC state to the partially synchronous periodic state (PSP) of structure $a b c b a d$ (still a very small probability exists for the system to approach the $a b b a c c$ structure if noise is very
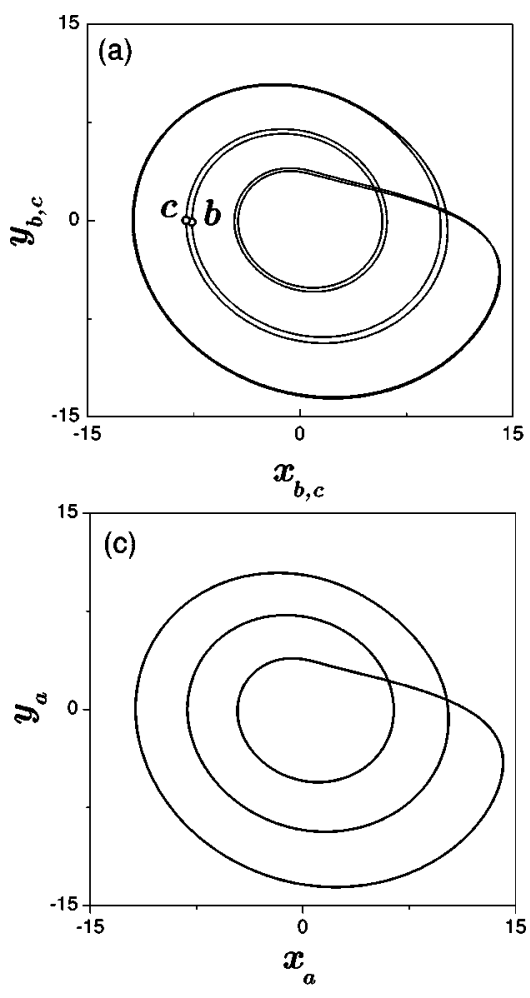
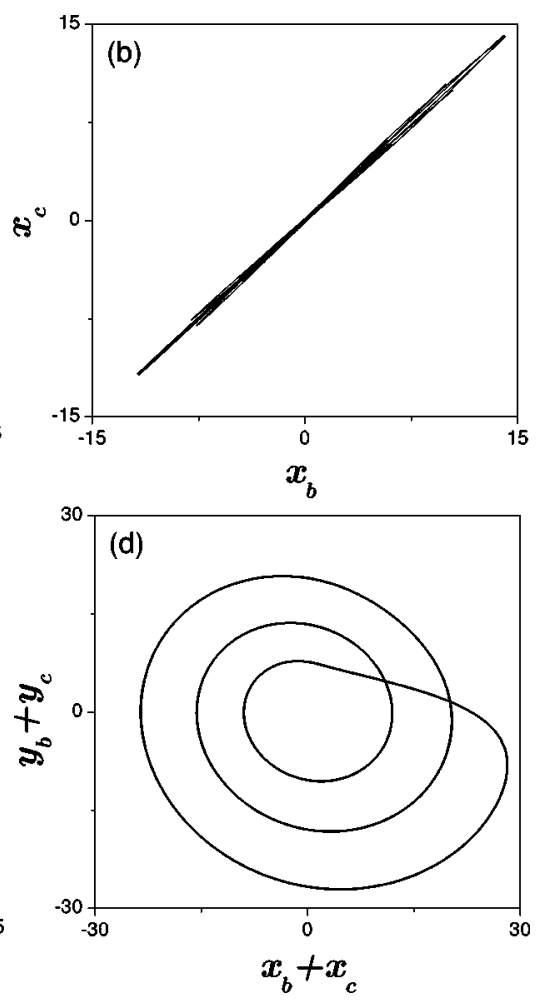

FIG. 6. Periodic oscillation in the pattern of $\{a b b a c c\}$ with $\varepsilon=0.007$. (a) $x_{b, c}$ vs $y_{b, c}$. (b) $x_{b}$ vs $x_{c}$. (c) $x_{a}$ vs $y_{a}$. (d) $x_{b}+x_{c}$ vs $y_{b}+y_{c}$. 

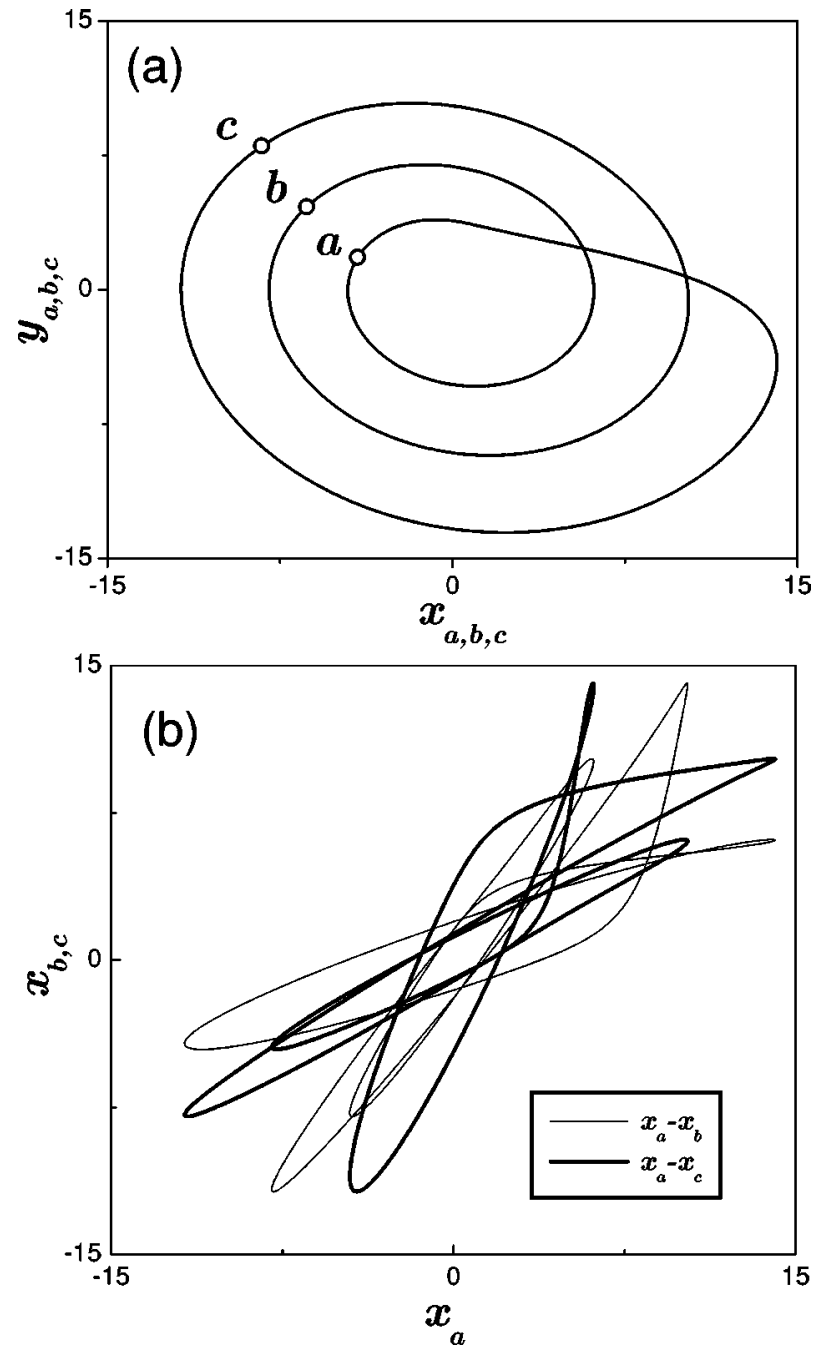

FIG. 7. Periodic oscillation in the pattern of $\{a b c a b c\}$ with $\varepsilon=0.005$. (a) $x_{a, b, c}$ vs $y_{a, b, c}$. (b) $x_{a}$ vs $x_{b, c}$.

small; this branch is not shown in Fig. 9). Further reducing $\varepsilon$, the abcbad PSP state is replaced by a partially synchronous quasiperiodic state (PSQ) via Hopf bifurcation with the same spatial $a b c b a d$ structure at $\varepsilon=0.067$. A bifurcation of Ruelle-Taken-type occurs at $\varepsilon \approx 0.056$; then this PSQ bifurcates to the PSC chaotic abcbad state. After $\epsilon<0.048$, the branch of stable PS solution (Fig. 9) stops to exist; the system jumps to the state of desynchronization abcdef chaotic structure via on-off intermittency between the identical sites of PSC. By continually decreasing $\varepsilon$, this desynchronous chaos may be interrupted by two periodic windows, one $a b a b a b$ PSP and the other $a a b a a b$ and $a b c a b c$ PSP via saddle-node bifurcation, and finally returns back to the fully desynchronous chaos till $\varepsilon=0$.

\section{CONCLUSION}

In conclusion we have investigated partially synchronous states of coupled chaotic Rossler oscillators in detail. We have revealed very rich spatial structures for the partial synchronization. The overall demonstration about the distribution of various stable PS and CS states is presented in Fig.

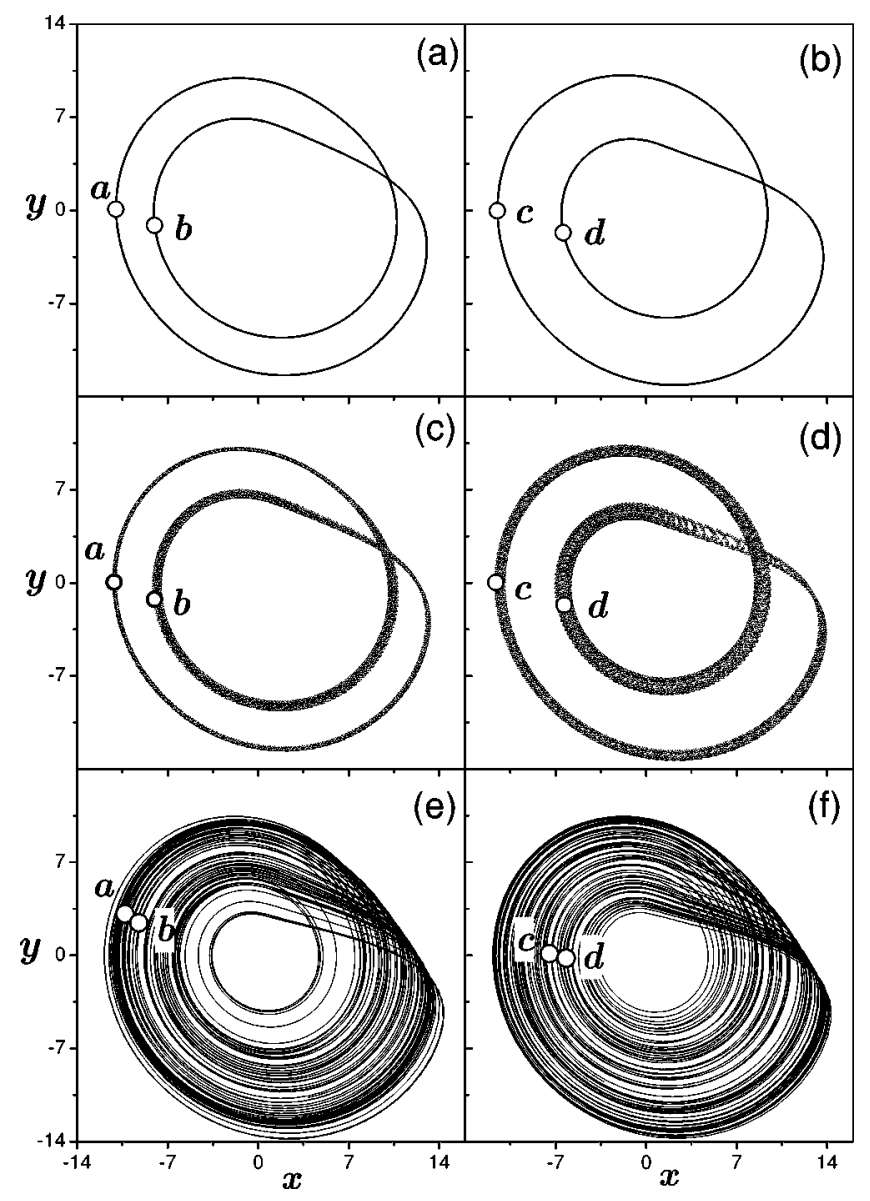

FIG. 8. (a) and (b) Periodic oscillation in the pattern of $\{a b c b a d\}$ with $\varepsilon=0.085$. (a) $x_{a, b}$ vs $y_{a, b}$. (b) $x_{c, d}$ vs $y_{c, d}$. (c) and (d) Quasiperiodic oscillation in the pattern of $\{a b c b a d\}$ with $\varepsilon$ $=0.065$. (c) $x_{a, b}$ vs $y_{a, b}$. (d) $x_{c, d}$ vs $y_{c, d}$. (e) and (f) Chaotic oscillation in the pattern of $\{a b c b a d\}$ with $\varepsilon=0.053$. (e) $x_{a, b}$ vs $y_{a, b}$. (f) $x_{c, d}$ vs $y_{c, d}$.

10 (a), where the state $\{a b c d e f\}$ represents the entirely desynchronous oscillators. Different spatial structures represent distinctive spatial symmetry breakings and retain different symmetries. For instance, the $\{a b a b a b\}$ structure breaks the symmetry of $x_{i} \rightarrow x_{i+1}$, but it keeps the symmetry of $x_{i}$ $\rightarrow x_{i+2}$; moreover, it still keeps the orientation symmetry (i.e., the state is invariant between the clockwise and counterclockwise orientations). The $\{a a b a a b\}$ and $\{a b c a b c\}$ states break the symmetries of $x_{i} \rightarrow x_{i+1}$ and $x_{i} \rightarrow x_{i+2}$, but still keep the symmetry of $x_{i} \rightarrow x_{i+3}$. However, the $\{a a b a a b\}$ state keeps the orientation symmetry while $\{a b c a b c\}$ does not. The states $\{a b b a c c\}$ and $\{a b c b a d\}$ break all translation symmetries for $x_{i} \rightarrow x_{i+j}, j=1,2,3,4,5$, but both keep the orientation symmetry.

It is emphasized that the above classifications can be degenerated in certain regions. For instance, all PS states except the $\{a b c b a d\}$ state reduce to the stable CSC $\{a a a a a a\}$ state by degenerating $a=b=c$ for $\varepsilon>0.087$. The $\{a b c b a d\}$ $(m=4)$ state can degenerate to $\{$ aacaac $\}(m=2)$ and $\{a b a b a b\}(m=2)$ states by setting $a=b, c=d$, and $c=a$, $d=b$, respectively. The $\{a b c a b c\},\{a b b a c c\}$ states can degenerate to $\{a b b a b b\}$ state. Similar degenerating processes 


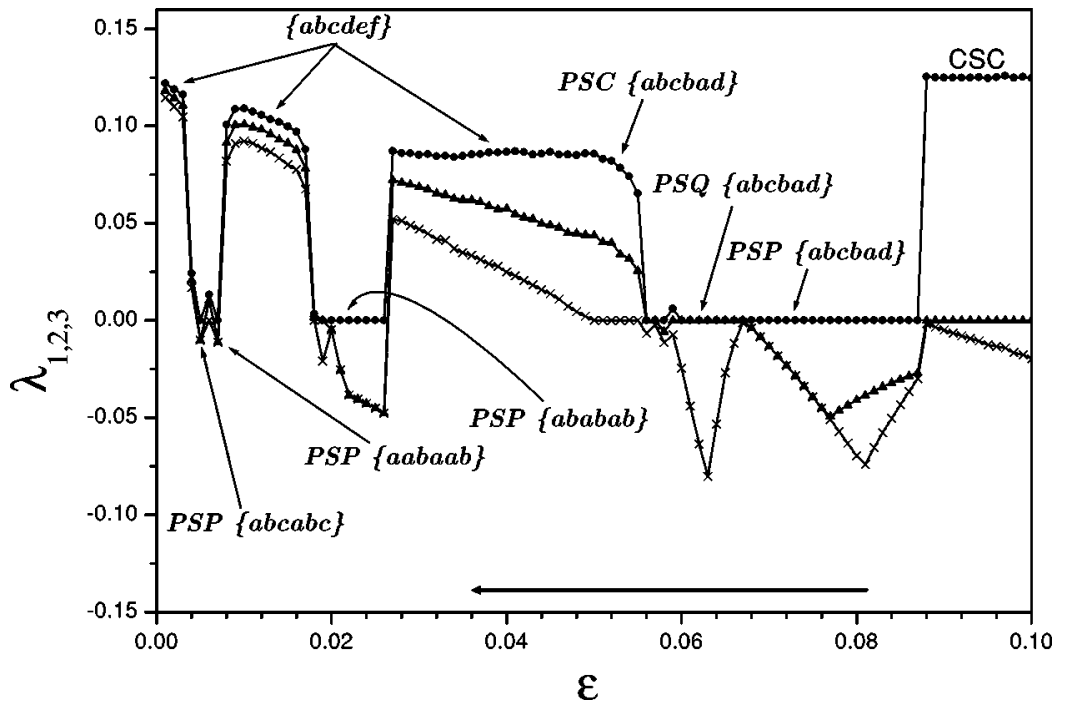

FIG. 9. The largest three Lyapunov exponents vs $\epsilon$. We start from $\epsilon>\epsilon_{c}$. SC, synchronous chaos; PSC, partially synchronous chaos; PSP, partially synchronous periodic motion; PSQ, partially synchronous quasiperiodic motion. from large $m$ to small $m$ can be observed for other structures. In Fig. 10(b), we represent all degenerated patterns in Fig. 10 (a) by the pattern with the least $m$; some patterns with large $m$ in (a) disappear in (b).

It is also emphasized that the above partially synchronous states can be easily observed in the original coupled system. For instance, in the coupling regions $0.020<\varepsilon<0.025$ and $0.050<\varepsilon<0.087$, we can observe only the PS state in Eqs. (1) from arbitrary initial conditions. The transitions from completely desynchronous chaos to PS states correspond to distinctive collapses of the system state from highdimensional variable space to different low-dimensional invariant subspaces. Moreover, the temporal behaviors of PS states are also very rich. The states can be periodic, quasiperiodic or chaotic, depending on parameter combinations [see Figs. 9 and 10].

In this paper, we focus on a particular model of coupled Rossler oscillators with $N=6$ only for the specification of demonstration. Similar analysis can be applied to different $N$, and similar PS states can be found identically. The applica-
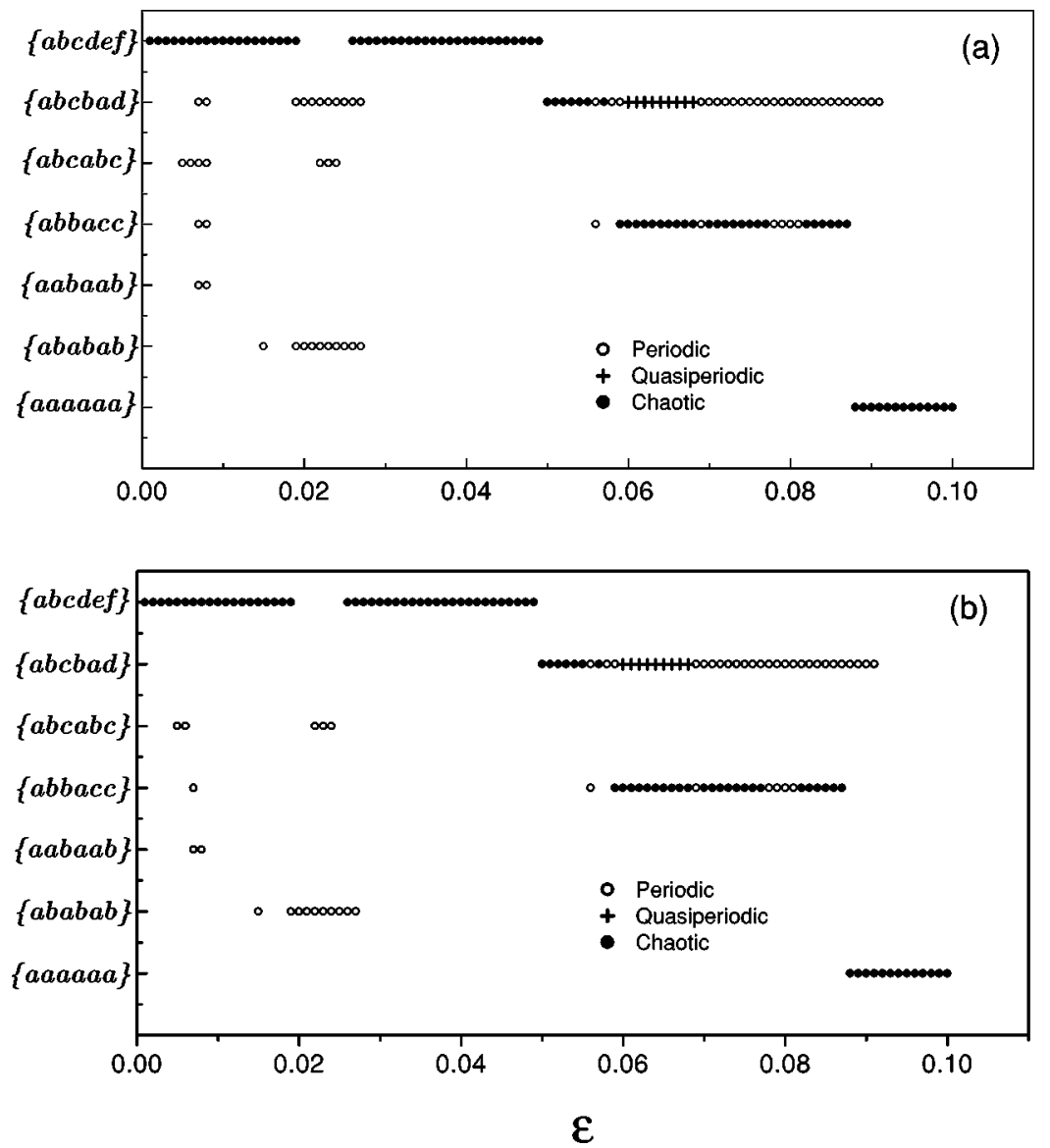

FIG. 10. (a) Overall demonstration of the system dynamics. The state $\{a b c d e f\}$ represents the entirely desynchronous state. (b) The same as (a) with the degenerated patterns with large $m$ being canceled. 
tions of the study to other coupled identical chaotic oscillators are also straightforward.

\section{ACKNOWLEDGMENTS}

This work was supported by the National Science Foundation of China, the Special Funds for Major State Basic Research Project, and Doctoral Program Foundation of Institute of Higher Education.

\section{APPENDIX}

Given the $\{a b b a c c\}$ state, the $3 \times 3 D$ invariant submanifold is defined by $\vec{r}_{1}=\vec{r}_{4}, \vec{r}_{2}=\vec{r}_{3}$, and $\vec{r}_{5}=\vec{r}_{6}$. By the transformation

$$
\begin{gathered}
\vec{R}_{1}=\vec{r}_{1}+\vec{r}_{4}, \quad \vec{R}_{2}=\vec{r}_{2}+\vec{r}_{3}, \\
\vec{R}_{3}=\vec{r}_{5}+\vec{r}_{6}, \quad \vec{R}_{4}=\vec{r}_{1}-\vec{r}_{4}, \\
\vec{R}_{5}=\vec{r}_{2}-\vec{r}_{3}, \quad \vec{R}_{6}=\vec{r}_{5}-\vec{r}_{6}, \\
\vec{R}_{i}=\left(X_{i}, Y_{i}, Z_{i}\right), \quad i=1,2, \ldots, N,
\end{gathered}
$$

we can derive nine equations

$$
\begin{gathered}
\dot{X}_{1}=-Y_{1}-Z_{1}+\varepsilon\left(X_{2}+X_{3}-2 X_{1}\right), \\
\dot{Y}_{1}=X_{1}+a Y_{1}+\varepsilon\left(Y_{2}+Y_{3}-2 Y_{1}\right), \\
\dot{Z}_{1}=2 b+\frac{1}{2} Z_{1} X_{1}-c Z_{1}+\varepsilon\left(Z_{2}+Z_{3}-2 Z_{1}\right), \\
\dot{X}_{2}=-Y_{2}-Z_{2}+\varepsilon\left(X_{1}-X_{2}\right), \\
\dot{Y}_{2}=X_{2}+a Y_{2}+\varepsilon\left(Y_{1}-Y_{2}\right), \\
\dot{Z}_{2}=2 b+\frac{1}{2} Z_{2} X_{2}-c Z_{2}+\varepsilon\left(Z_{1}-Z_{2}\right), \\
\dot{X}_{3}=-Y_{3}-Z_{3}+\varepsilon\left(X_{1}-X_{3}\right), \\
\dot{Y}_{3}=2 b+\frac{1}{2} Z_{3} X_{3}-c Z_{3}+\varepsilon\left(Z_{1}-Z_{3}\right), \\
\end{gathered}
$$

for the motion on the synchronous invariant submanifold, and determine the stability of the given partially synchronized pattern by the set of equations below:

$$
\begin{gathered}
\delta \dot{X}_{4}=-\delta Y_{4}-\delta Z_{4}+\varepsilon\left(\delta X_{5}-\delta X_{6}-2 \delta X_{4}\right) \\
\delta \dot{Y}_{4}=\delta X_{4}+a \delta Y_{4}+\varepsilon\left(\delta Y_{5}-\delta Y_{6}-2 \delta X_{4}\right) \\
\delta \dot{Z}_{4}=\frac{1}{2} Z_{1} \delta X_{4}+\frac{1}{2} X_{1} \delta Z_{4}-c \delta Z_{4}+\varepsilon\left(\delta Z_{5}-\delta Z_{6}-2 \delta Z_{4}\right) \\
\delta \dot{X}_{5}=-\delta Y_{5}-\delta Z_{5}+\varepsilon\left(\delta X_{4}-3 \delta X_{5}\right) \\
\delta \dot{Y}_{5}=\delta X_{5}+a \delta Y_{5}+\varepsilon\left(\delta Y_{4}-3 \delta Y_{5}\right) \\
\delta \dot{Z}_{5}=\frac{1}{2} Z_{2} \delta X_{5}+\frac{1}{2} X_{2} \delta Z_{5}-c \delta Z_{5}+\varepsilon\left(\delta Z_{4}-3 \delta Z_{5}\right) \\
\delta \dot{X}_{6}=-\delta Y_{6}-\delta Z_{6}+\varepsilon\left(-3 \delta X_{6}-\delta X_{4}\right) \\
\delta \dot{Y}_{6}=\delta X_{6}+a \delta Y_{6}+\varepsilon\left(-3 \delta Y_{6}-\delta Y_{4}\right) \\
\delta \dot{Z}_{6}=\frac{1}{2} Z_{3} \delta X_{6}+\frac{1}{2} X_{3} \delta Z_{6}-c \delta Z_{6}+\varepsilon\left(-3 \delta Z_{6}-\delta Z_{4}\right)
\end{gathered}
$$

The evolution equations and stability equations for $\{a b c a b c\}$ patterns can be specified in a similar manner. We will not repeat the details.

For $m=4$, only one possible pattern exists; it is $\{a b c b a d\}$. Now the transformation

$$
\begin{gathered}
\vec{R}_{1}=\vec{r}_{1}+\vec{r}_{5}, \quad \vec{R}_{2}=\vec{r}_{2}+\vec{r}_{4}, \\
\vec{R}_{3}=\vec{r}_{3}, \quad \vec{R}_{4}=\vec{r}_{6}, \\
\vec{R}_{5}=\vec{r}_{1}-\vec{r}_{5}, \quad \vec{R}_{6}=\vec{r}_{2}-\vec{r}_{4}, \\
\vec{R}_{i}=\left(X_{i}, Y_{i}, Z_{i}\right), \quad i=1,2, \ldots, N,
\end{gathered}
$$

which leads to the evolution equations

$$
\begin{gathered}
\dot{X}_{1}=-Y_{1}-Z_{1}+\varepsilon\left(2 X_{4}+X_{2}-2 X_{1}\right), \\
\dot{Y}_{1}=X_{1}+a Y_{1}+\varepsilon\left(2 Y_{4}+Y_{2}-2 Y_{1}\right), \\
\dot{Z}_{1}=2 b+\frac{1}{2} Z_{1} X_{1}-c Z_{1}+\varepsilon\left(2 Z_{4}+Z_{2}-2 Z_{1}\right), \\
\dot{X}_{2}=-Y_{2}-Z_{2}+\varepsilon\left(X_{1}+2 X_{3}-2 X_{2}\right), \\
\dot{Y}_{2}=X_{2}+a Y_{2}+\varepsilon\left(Y_{1}+2 Y_{3}-2 Y_{2}\right), \\
\dot{Z}_{2}=2 b+\frac{1}{2} Z_{2} X_{2}-c Z_{2}+\varepsilon\left(Z_{1}+2 Z_{3}-2 Z_{2}\right), \\
\dot{X}_{3}=-Y_{3}-Z_{3}+\varepsilon\left(X_{2}-2 X_{3}\right), \\
\dot{Y}_{3}=X_{3}+a Y_{3}+\varepsilon\left(Y_{2}-2 Y_{3}\right), \\
\dot{Z}_{3}=b+Z_{3} X_{3}-c Z_{3}+\varepsilon\left(Z_{2}-2 Z_{3}\right), \\
\dot{X}_{4}=-Y_{4}-Z_{4}+\varepsilon\left(X_{1}-2 X_{4}\right), \\
\dot{Y}_{4}=X_{4}+a Y_{4}+\varepsilon\left(Y_{1}-2 Y_{4}\right), \\
\dot{Z}_{4}=b+Z_{4} X_{4}-c Z_{4}+\varepsilon\left(Z_{1}-2 Z_{4}\right),
\end{gathered}
$$


in the submanifold and the transverse stability is determined by

$$
\begin{gathered}
\delta \dot{X}_{5}=-\delta Y_{5}-\delta Z_{5}+\varepsilon\left(\delta X_{6}-2 \delta X_{5}\right), \\
\delta \dot{Y}_{5}=\delta X_{5}+a \delta Y_{5}+\varepsilon\left(\delta Y_{6}-2 \delta Y_{5}\right), \\
\delta \dot{Z}_{5}=\frac{1}{2} Z_{1} \delta X_{5}+\frac{1}{2} X_{1} \delta Z_{5}-c \delta Z_{5}+\varepsilon\left(\delta Z_{6}-2 \delta Z_{5}\right),
\end{gathered}
$$

[1] L. M. Pecora and T. L. Carroll, Phys. Rev. Lett. 64, 821 (1990); Phys. Rev. A 44, 2374 (1991); J. F. Heagy, T. L. Carroll, and L. M. Pecora, Phys. Rev. E 50, 1874 (1994); R. Brown and N. F. Rulkov, Phys. Rev. Lett. 78, 4189 (1997); G. Hu, J. Z. Yang, W. Q. Ma, and J. H. Xiao, ibid. 81, 5314 (1998); P. Shi, G. Hu, and L. Xu, Acta Phys. Sin. 49, 24 (2000).

[2] L. Fabiny, P. Colet, and R. Roy, Phys. Rev. A 47, 4287 (1993); R. Roy and K. S. Thornburg, Jr., Phys. Rev. Lett. 72, 2009 (1994); S. K. Han, C. Kurrer, and Y. Kuramoto, ibid. 75, 3190 (1995); D. J. Gauthier and J. C. Bienfang, ibid. 77, 1751 (1996); V. Astakhov, A. Shabunin, T. Kapitaniak, and V. Anishchenko, ibid. 79, 1014 (1997).

[3] C. M. Gray, P. Koenig, A. K. Engel, and W. Singer, Nature (London) 338, 334 (1989); K. Kaneko, in Proceedings of the 19th IUPAP International Conference on Statistical Physics, Xiamen, China, edited by Bai-Lin Hao (World Scientific, Singapore, 1996), p. 338.

$$
\delta \dot{X}_{6}=-\delta Y_{6}-\delta Z_{6}+\varepsilon\left(\delta X_{5}-2 \delta X_{6}\right),
$$

$$
\delta \dot{Y}_{6}=\delta X_{6}+a \delta Y_{6}+\varepsilon\left(\delta Y_{5}-2 \delta Y_{6}\right),
$$

$\delta \dot{Z}_{6}=\frac{1}{2} Z_{2} \delta X_{6}+\frac{1}{2} X_{2} \delta Z_{6}-c \delta Z_{6}+\varepsilon\left(\delta Z_{5}-2 \delta Z_{6}\right)$.
[4] K. Pyragas, Phys. Rev. E 54, R4508 (1996); M. Inoue, T. Kawazoe, Y. Nishi, and M. Nagadome, Phys. Lett. A 249, 69 (1998); M. Hasler, Y. Maistrenko, and O. Popovych, Phys. Rev. E 58, 6843 (1998).

[5] O. E. Rossler, Phys. Lett. 57A, 397 (1976).

[6] G. Hu, Y. Zhang, H. A. Cerdeira, and S. G. Chen, Phys. Rev. Lett. 85, 3377 (2000).

[7] E. Ott and J. C. Sommerer, Phys. Lett. A 188, 39 (1994).

[8] J. F. Heagy, L. M. Pecora, and T. L. Carroll, Phys. Rev. Lett. 74, 4185 (1995); M. Ding and W. Yang, Phys. Rev. E 54, 2489 (1996); J. Yang, G. Hu, and J. Xiao, Phys. Rev. Lett. 80, 496 (1998); Y. L. Maistrenko, V. L. Maitrenko, A. Popovich, and E. Mosekilde, Phys. Rev. E 57, 2713 (1998); Phys. Rev. Lett. 80, 1638 (1998); G. Hu, F. Xie, Z. Qu, and P. Shi, Commun. Theor. Phys. 31, 99 (1999); Y. L. Maistrenko, V. L. Maitrenko, O. Popovich, and E. Mosekilde, Phys. Rev. E 60, 2817 (1999). 